\title{
Michel Pernot (dir.), Quatre mille ans d'histoire du cuivre, fragments d'une suite de rebonds
}

Bordeaux, Presses universitaires de Bordeaux, collection « THEA », 2017, $360 \mathrm{p}$.

\section{Julien Flament}

\section{(2) OpenEdition}

Édition électronique

URL : http://journals.openedition.org/artefact/3706

DOI : 10.4000/artefact.3706

ISSN : 2606-9245

Éditeur :

Association Artefact. Techniques histoire et sciences humaines, Presses universitaires du Midi

Édition imprimée

Date de publication : 15 mars 2019

Pagination : 333-338

ISBN : 978-2-8107-0623-5

ISSN : 2273-0753

Référence électronique

Julien Flament, « Michel Pernot (dir.), Quatre mille ans d'histoire du cuivre, fragments d'une suite de rebonds », Artefact [En ligne], 9 | 2018, mis en ligne le 04 mars 2020, consulté le 29 novembre 2020 URL : http://journals.openedition.org/artefact/3706 ; DOI : https://doi.org/10.4000/artefact.3706

Ce document a été généré automatiquement le 29 novembre 2020.

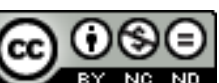

Artefact, Techniques, histoire et sciences humaines est mise à disposition selon les termes de la Licence Creative Commons Attribution - Pas d'Utilisation Commerciale - Pas de Modification 4.0 International. 


\title{
Michel Pernot (dir.), Quatre mille ans d'histoire du cuivre, fragments d'une suite de rebonds
}

Bordeaux, Presses universitaires de Bordeaux, collection « THEA », 2017, $360 \mathrm{p}$.

\author{
Julien Flament
}

1 L'ouvrage édité par Michel Pernot est consacré au cuivre et à ses alliages sur une période de quatre millénaires qui s'étend de la découverte de la métallurgie jusqu'à l'usage des produits cuivreux dans les technologies les plus récentes. C'est en réalité une histoire discontinue dont plusieurs « fragments » sont présentés dans cet ouvrage sous la forme de « rebonds".

2 «Comment et pourquoi produire des objets en alliages cuivreux?» (p. 9). La diversité des réponses apportées à cette problématique reflète la sensibilité des différents contributeurs - qu'ils soient historiens, archéologues, archéomètres ou artisans - et donne une réelle dimension interdisciplinaire à l'ouvrage. La première partie introductive est formée de trois textes qui fixent le cadre et les enjeux de l'étude. Dans les deux premiers, Michel Pernot insiste sur le lien étroit qui unit «les domaines du matériel et de l'immatériel » (p.17) que sont la métallurgie du cuivre et les structures sociales des sociétés anciennes. Les difficultés des recherches sur la place du cuivre dans ces sociétés ne sont pas occultées, en particulier la question du recyclage, son ampleur et par conséquent la représentativité des mobiliers qui nous sont parvenus presque par accident. L'introduction se termine par une contribution de Paolo Piccardo, Justine Vernet et Giorgia Ghiara qui aborde les propriétés physiques du cuivre et de ses alliages. Le propos, très didactique et utile aux non spécialistes, est accompagné de nombreux exemples qui démontrent les apports majeurs des sciences des matériaux pour la compréhension des pratiques métallurgiques anciennes.

La suite de l'ouvrage est organisée de manière chronologique avec une deuxième partie qui recouvre "une large Antiquité » et brasse des cultures variées depuis l'Europe occidentale jusqu'au Proche-Orient. Avec cinq contributions, cette période marquée par 
la découverte puis la maturation de la métallurgie du cuivre et de ses alliages occupe une place significative dans l'ouvrage qui illustre la vitalité des recherches en cours. Dans un premier texte, Michel Pernot dresse un état des connaissances sur l'origine de la métallurgie et les conditions qui ont permis son émergence (maîtrise du feu, connaissance de la nature des roches). Il questionne le lien entre l'essor des pratiques métallurgiques et celui des sociétés hiérarchisées, mais également le rôle et la place qu'y occupe le métallurgiste. Ce dernier ne doit pas être considéré comme un artisan qui travaille de manière isolée: il est en interaction permanente avec d'autres spécialistes comme le potier pour la confection des moules, des creusets, ou encore des structures de chauffe. De la même manière, la synergie entre le verrier et le métallurgiste mériterait d'être davantage explorée. Le texte suivant de Ziad El Morr se concentre sur les pratiques métallurgiques des artisans du Proche-Orient à partir de quatre sites qui couvrent les trois derniers millénaires avant notre ère. Les analyses archéométriques que l'auteur a mises en œuvre permettent de déterminer la composition élémentaire du mobilier à base de cuivre et de restituer les recettes dont il propose une classification selon leurs caractères économique, prestigieux ou fonctionnel. Il démontre ainsi les multiples enjeux à l'origine des choix des métallurgistes qui ne se réduisent pas simplement à l'optimisation des propriétés physiques des matériaux et des étapes de la chaîne opératoire mais intègrent également des critères esthétiques, sociaux et traditionnels. L'usage du laiton ou encore le choix de subsister l'étain à l'arsenic pour la production de «bronzes » en constituent des exemples éloquents. Ces résultats trouvent un prolongement dans la contribution d'Anne Lehoërff qui explore le lien entre le métal et la guerre dans l'Europe protohistorique. Alors que les armes en alliages cuivreux procédaient jusqu'alors de copies de productions en pierre, l'auteur met en évidence les innovations au service de la guerre dans les productions métallurgiques du $\mathrm{II}^{\mathrm{e}}$ millénaire. Il s'agit notamment de la diversification fonctionnelle de certains outils, tels que la hache, voire de la création d'objets à la fonction exclusivement guerrière comme l'épée. Le décloisonnement entre les métallurgies du cuivre et du fer, amorcé dans la contribution d'Anne Lehoërff, se poursuit avec Alexis Gorgues qui s'intéresse à la période de transition entre les âges du Bronze et du Fer en Europe occidentale et méditerranéenne. Ainsi, la proximité entre le travail du bronzier et celui du forgeron est discutée à l'aune de la fouille de la Tossal Montanes (province de Teruel, Espagne). Alexis Gorgues évoque les similarités des chaînes opératoires : déformation, recuit, trempe et emploi de structures de chauffe semblables. Il nous semble que cette interpénétration doit être également envisagée dans le champ de la métallurgie extractive pour discuter dans quelle mesure le traitement de minerais polymétalliques de cuivre et de fer - tels que la chalcopyrite - a pu constituer une étape préliminaire à la maîtrise de la réduction des minerais de fer. Dans l'ultime contribution de cette partie concernant une «large Antiquité », Michel Pernot s'intéresse aux évolutions de la culture technique du cuivre au cours du I ${ }^{\text {er }}$ millénaire avant notre ère. Une grande stabilité marque sa première moitié avant que ne soient introduites plusieurs innovations, notamment la production par fonderie de pièces en creux (qui nécessitent l'usage d'un noyau), l'introduction du procédé de fonte à la cire perdue ou encore la technique du bronzage de pièces en fer.

4 Avec seulement un "fragment» abordé parmi cette longue histoire du cuivre, la période médiévale fait figure de parent pauvre de la recherche sur la métallurgie du cuivre et de ses alliages. La contribution de Nicolas Thomas s'attache à définir les modalités de production des objets à base de cuivre pour le haut Moyen Âge et le 
Moyen Âge tardif. Les distinctions très nettes entre ces deux périodes concernent tant les techniques de mise en forme que les volumes ou les destinations des productions. À la confection sur commande de petits objets des temps mérovingiens succèdent à partir du XIII ${ }^{e}$ siècle les productions de masse marquées par la spécialisation croissante des artisans et la concentration des activités dans quelques grands centres.

5 L'étude de Jean-François Moreau sur l'échange de chaudrons de cuivre entre l'Europe et l'Amérique du Nord est l'unique contribution qui couvre la période moderne. Si les études archéométriques ont permis de mettre en évidence l'usage du cuivre natif par les Amérindiens ${ }^{1}$, l'auteur montre l'incidence sur leurs pratiques sociales de l'arrivée des chaudrons de cuivre européens. Les recherches archéologiques permettent de suivre la diffusion de ces produits dans l'espace nord-américain et les usages qu'en font les Amérindiens, qu'ils soient culinaires ou sépulturaux.

6 La période contemporaine, celle des révolutions industrielles, est l'occasion grâce à la contribution d'Alexandre Fernandez de s'intéresser à la métallurgie extractive à travers l'étude du cas de la région métallurgique galloise de Swansea. Au milieu du XIX siècle, l'émergence de ce pôle de production de premier plan s'explique par la combinaison de ressources en combustibles, d'une maîtrise technique (avec l'introduction de four à réverbère) et de capitaux. Alexandre Fernandez montre que Swansea n'est que le point de départ de l'essor de la production du cuivre dont le prolongement se trouve en Amérique du Nord à partir de la fin du XIX ${ }^{e}$ siècle. Maryse Lassalle, Corinne Arvieu et Florent Laroche se penchent sur le rôle déterminant qu'a joué le cuivre dans le domaine énergétique au cours de la première révolution industrielle. En effet, la mise au point de machines à vapeur de fortes puissances est le fruit d'une bonne maitrise de la pression permise par le développement de chaudières en cuivre performantes, capables de résister à des contraintes mécaniques. Le cuivre occupe également une place majeure au cours des deuxième et troisième révolutions industrielles comme le montre ensuite Christophe Bouneau. S'il s'est trouvé rapidement supplanté par l'aluminium lors du grand mouvement d'électrification initié à partir de la fin du xix siècle, il a conservé de nombreuses applications grâce à sa meilleure conductivité électrique. Ce «matériau polymorphe d'innovations» (p. 251), omniprésent à l'ère des télécommunications, est l'un des enjeux de la transition énergétique du xxi ${ }^{e}$ siècle.

7 La cinquième section de l'ouvrage abandonne l'ordre chronologique pour aborder des thématiques aux carrefours de l'art et de l'industrie. Jean-Marie Welter livre d'abord une étude détaillée des procédés industriels de laminage et de coulée continue. Il s'appuie sur la notion de système technique, introduite par Bertrand Gille ${ }^{2}$, pour expliquer l'émergence de la coulée continue au moment où les capacités de laminage s'accroissent grâce à l'électricité puis à l'électronique. Dans la contribution suivante de Jean Dubos, c'est le point de vue du praticien, du fondeur d'art, qui permet au lecteur d'entrer dans la réalité pratique du travail du cuivre et de ses alliages. Si l'importance du geste et de l'outil est capitale pour restituer les chaînes opératoires, les expérimentations conduites par Jean Dubos mettent également en exergue l'appréciation sensorielle à travers la manière dont "sonne " le métal au cours du martelage ainsi que sa couleur à la chauffe. Enfin, la dernière contribution de Valeria Bongiorno, Justine Vernet et Paolo Piccardo se concentre sur l'étude de la statuaire du cimetière génois de Staglieno à travers la problématique de la conservation et de la restauration de la statuaire funéraire à base de cuivre. La caractérisation des patines présentes sur ce mobilier apporte des informations précieuses pour définir les 
stratégies de préservation et distinguer ce qui relève du parti pris originel de l'artiste ou de l'altération.

Dans un style très différent, les deux dernières contributions ne prétendent pas recoller l'ensemble des «fragments » de cette longue histoire du cuivre. Pour Anne-Françoise Garçon, la longévité et l'omniprésence du cuivre dans les sociétés anciennes sont l'occasion d'entreprendre une réflexion sur les bouleversements matériels mais également intellectuels induits par le cuivre et ses alliages au sein des sociétés humaines. Quant à l'ultime contribution de Michel Pernot, elle dresse un panorama du métal rouge dans les sociétés passées tout en proposant, en guise d'ouverture, d'élargir les recherches sur l'usage du cuivre au domaine de la métrologie et de son mobilier particulier.

9 Les textes rassemblés dans cet ouvrage sont bien écrits, clairs et les illustrations choisies viennent judicieusement à l'appui de la démonstration bien qu'on regrette l'absence d'échelle sur plusieurs photographies de mobilier archéologique. Sur le fond, la discrétion de la métallurgie extractive, c'est-à-dire les étapes de la transformation du minerai en métal, est notable et assumée par Michel Pernot. Il s'agit pourtant d'un champ de la recherche archéo-métallurgique qui s'est fortement renouvelé ces dernières années et dont les résultats permettent d'explorer plusieurs aspects des productions à base de cuivre, notamment l'incidence de la disponibilité des ressources minérales sur les alliages produits et les techniques employées ${ }^{3}$. Par ailleurs, et bien que l'historiographie puisse expliquer la place prépondérante des périodes anciennes au sein de l'ouvrage, la discrétion des périodes médiévale et moderne est à déplorer d'autant plus que des travaux récents éclairent plusieurs aspects de la production et de la consommation du cuivre et de ses alliages à travers l'étude archéométrique du mobilier ${ }^{4}$. De la même manière, l'examen des sources écrites a permis d'identifier de nouveaux procédés d'obtention du métal rouge à partir de minerais polymétalliques complexes dont l'exploitation modifie profondément et durablement le marché du cuivre de l'Europe moderne ${ }^{5}$.

Certes nous ne proposons ni plus ni moins que d'autres «fragments » de l'histoire du cuivre et de ses alliages. L'ouvrage en livre déjà suffisamment pour permettre au lecteur de saisir l'importance de ce matériau dans les sociétés, qu'elles soient passées ou présentes. Surtout, cette synthèse diachronique convoque de nombreuses disciplines dont les spécialistes ont démontré la nécessité d'une approche, historique, archéologique, archéométrique et pratique du métal rouge.

\section{NOTES}

1. Laure Dussubieux, Aurélie DERAISME, Gérard FROT, Christopher STEVENSON, Amy CREECH et Yves BIENVENU, «LA-ICP-MS, SEM-EDS and EPMA Analysis of Eastern North American Copper-Based Artefacts: Impact of Corrosion and Heterogeneity on the Reliability of the LA-ICP-MS Compositional Results ", Archaeometry, n 50, 2008, p. 643-657 ; Mark A. HILL, Diana M. GREENLEE et 
Henry H. NEFF, "Assessing the provenance of Poverty Point copper through LA-ICP-MS compositional analysis ", Journal of Archaeological Science: Reports, $n^{\circ}$ 6, 2006, p. 351-360.

2. Bertrand GILLE (dir.), Histoire des techniques. Technique et civilisations, technique et sciences, Paris, Gallimard, 1978.

3. Georges VERLY, "The Smelting Furnaces of Ayn Soukhna: The Excavations of 2013, 2014 and 2015 ", in Ignacio MONTERO RUIZ et Alicia PEREA (dir.), Archaeometallurgy in Europe IV, Actes du colloque du $1^{\text {er }}$-3 juin 2015, Madrid, Bibliotheca Praehistorica Hispana, vol. xxxIII, 2007, p. 143-157; Bastian ASmus, Medieval Copper Smelting in the Harz Mountains, Germany, Bochum, Deutsches Bergbau-Museum, 2012.

4. Manon CASTELLE, Les techniques de fabrication de la grande statuaire en bronze 1540-1660 en France, Thèse de $3^{\mathrm{e}}$ cycle, Université de Versailles-Saint-Quentin-en-Yvelines, 2016; Stephen MERKEL, «Carolingian and Ottonian Brass Production in Westphalia. Evidence from the Crucibles and Slag of Dortmund and Soest ", Metalla, $\mathrm{n}^{\circ} 22-1,2016$, p. 21-39; Olivier THUAUDET, Les accessoires métalliques du vêtement et de la parure de corps en Provence du $\mathrm{XI}^{e}$ au XVI ${ }^{e}$ siècle : étude archéologique et approche croisée d'une production méconnue. Thèse de doctorat, Aix-Marseille Université, 2016.

5. Philippe BRAunstein, Les Allemands à Venise (1380-1520), Rome, École française de Rome, 2016, p. 534 et suivantes; Martin M. ELBL, « From Venice to the Tuat: Trans-Saharan Copper Trade and Francesco di Marco Datini of Prato », in Lawrin ARMSTRONG, Ivana ELBL et Martin M. ELBL (dir.), Money, Markets and Trade in Late Medieval Europe: Essays in Honour of John H. A. Munro, Leyde, Brill, 2007, p. 411-459.

\section{AUTEUR}

\section{JULIEN FLAMENT}

Chercheur associé IRAMAT-CEB UMR5060, CNRS/Université d'Orléans 